\title{
Influences on the start, selection and duration of treatment with antibiotics in long-term care facilities
}

\author{
Nick Daneman MD MSc, Michael A. Campitelli MPH, Vasily Giannakeas MPH, Andrew M. Morris MD SM(Epi), \\ Chaim M. Bell MD PhD, Colleen J. Maxwell PhD, Lianne Jeffs PhD, Peter C. Austin PhD, Susan E. Bronskill PhD
}

Cite as: CMAJ 2017 June 26;189:E851-60. doi: 10.1503/cmaj.161437

Visual abstract available at www.cmaj.ca/lookup/suppl/doi:10.1503/cmaj.161437/-/DC2

\begin{abstract}
BACKGROUND: Understanding the extent to which current antibiotic prescribing behaviour is influenced by clinicians' historical patterns of practice will help target interventions to optimize antibiotic use in long-term care. Our objective was to evaluate whether clinicians' historical prescribing behaviours influence the start, prolongation and class selection for treatment with antibiotics in residents of long-term care facilities.
\end{abstract}

METHODS: We conducted a retrospective cohort study of all physicians who prescribed to residents in long-term care facilities in Ontario between Jan. 1 and Dec. 31, 2014. We examined variability in antibiotic prescribing among physicians for 3 measures: start of treatment with antibiotics, use of prolonged durations exceeding 7 days and selection of fluoroquinolones. Funnel plots with control limits were used to determine the extent of variation and characterize physicians as extreme low, low, average, high and extreme high prescribers for each tendency. Multivariable logistic regression was used to assess whether a clinician's prescribing tendency in the previous year predicted current prescribing patterns, after accounting for residents' demographics, comorbidity, functional status and indwelling devices.

RESULTS: Among 1695 long-term care physicians, who prescribed for 93132 residents, there was wide variability in the start of antibiotic treatment (median $45 \%$ of patients, interquartile range [IQR] 32\%-55\%), use of prolonged treatment durations (median $30 \%$ of antibiotic prescriptions, IQR 19\%-46\%) and selection of fluoroquinolones (median 27\% of antibiotic prescriptions, IQR 18\%-37\%). Prescribing tendencies for antibiotics by physicians in 2014 correlated strongly with tendencies in the previous year. After controlling for individual resident characteristics, prior prescribing tendency was a significant predictor of current practice.

INTERPRETATION: Physicians prescribing antibiotics exhibited individual, measurable and historical tendencies toward start of antibiotic treatment, use of prolonged treatment duration and class selection. Prescriber audit and feedback may be a promising tool to optimize antibiotic use in long-term care facilities.

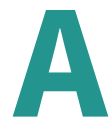

ntibiotics are among the most frequently prescribed drugs in long-term care facilities, ${ }^{1-3}$ and many prescriptions are inappropriate, with drugs prescribed for too long or poorly. ${ }^{4-6}$ As a result, vulnerable residents of these facilities are exposed to avoidable harms that range from allergy to organ-specific toxicities, ${ }^{4,7}$ Clostridium difficile infection ${ }^{8,9}$ and antimicrobial-resistant pathogens. ${ }^{10,11}$ Many harms affect not only the direct recipients of these drugs but also neighbouring and future residents of the facility. ${ }^{12}$

Antibiotic stewardship interventions in long-term care have had promising but inconsistent effects on antibiotic use. ${ }^{13-15}$ Prior initiatives have usually been multifaceted and have included educational sessions, ${ }^{16-21}$ development of local guidelines, ${ }^{16,17,21-23}$ portable reference material, ${ }^{16,19,23}$ on-site consultations about infectious diseases ${ }^{24}$ or academic detailing. ${ }^{18}$ Many of these interventions are labour intensive, and none have reported on cost-effectiveness or sustainability. ${ }^{13}$

In a previous study, we reported that the use of prolonged antibiotic treatment in long-term care is more dependent on who prescribes the drug than the characteristics of the resident receiving the drug. ${ }^{6}$ If decision-making about prescribing antibiotics is influenced by consistent and predictable historical patterns of practice - "prescriber tendencies" - then stewardship interventions focused on individual prescribers might offer an 
avenue to improve the use of antibiotics in long-term care facilities and reduce harms associated with overuse and misuse of these agents. In particular, peer comparison audit and feedback of prescriber profiles could be a pragmatic, effective and sustainable means of improving antimicrobial prescribing in long-term care on a broad scale. ${ }^{17,22,25}$

Therefore, in this province-wide study of long-term care physicians we examined 3 prescribing tendencies for antibiotics that span the range of decision-making about antibiotics: tendency to start treatment with antibiotics, tendency to use antibiotics for prolonged periods and tendency to select a specific class of antibiotics. In the absence of any large-scale initiatives for antibiotic stewardship, we hypothesized that these 3 prescribing tendencies would vary across prescribers more than would be expected by chance or explained by resident characteristics, and that a clinician's historical prescribing tendency would strongly predict their current prescribing practice to patients.

\section{Methods}

\section{Study design and data sources}

We conducted a retrospective cohort study of all physicians who prescribed to residents of long-term care facilities in Ontario between Jan. 1 and Dec. 31, 2014. We used data sets that were linked using encoded identifiers and analyzed at the Institute for Clinical Evaluative Sciences (ICES). Ontario is Canada's most populous province, with a population of 13 million. Each year, over 90000 of Ontario residents spend time in one of the province's more than 600 long-term care facilities (more than 70000 beds). ${ }^{12,26}$ The Ontario Ministry of Health and Long-Term Care provides universal health care coverage for residents of the province, including, but not limited to, physician fees, acute hospital care, long-term care, and drugs for those aged 65 years and over and/or in long-term care facilities. The administrative databases housing these data have been well validated in previous research, ${ }^{27,28}$ including extensive studies of antimicrobial use and $^{29,30}$ quality of long-term care,,$^{26,31}$ and confluence of these 2 fields - antimicrobial use in long-term care. , $12,32^{2}$

\section{Study population}

We identified all physicians who were responsible for prescribing to residents in long-term care facilities in Ontario during the study period by using the Ontario Drug Benefits Database, a comprehensive and accurate record of drugs dispensed to Ontario residents aged 65 years or older and all residents of long-term care facilites. ${ }^{33}$ We identified all physicians $(n=1869)$ who had written at least 100 long-term care prescriptions for any drug in both 2013 and 2014. The cut-off was chosen after reviewing the frequency distribution of long-term care prescriptions per physician. This volume of prescriptions was required to estimate prescribing tendencies with precision and to focus on physicians with large long-term care practices. We also excluded specialists who were not family physicians, geriatricians or internists (e.g., psychiatrists, neurologists, urologists) to remove those who were unlikely to be the primary physician responsible for prescribing treatment with antibiotics $(n=90)$; physicians who were missing an encoded physician number $(n=10)$ or physician characteristics $(n=66)$; or those with multiple physician numbers $(n=8)$. Our final cohort included 1695 physicians who regularly prescribed in long-term care facilities in both 2013 and 2014.

We identified all residents $(n=93132)$ with an assessment for long-term care in 2014 who were prescribed at least 1 drug (of any type) in 2014, by at least 1 of the physicians in the prescriber cohort.

\section{Prescribing tendencies for antibiotics}

We assessed 3 prescribing tendencies for antibiotics among longterm care prescribers: antibiotic initiation, use of prolonged treatment duration and selection of fluoroquinolones.

We calculated a prescriber's tendency to start treatment with antibiotics by dividing the number of unique residents in longterm care who were prescribed at least 1 antibiotic treatment by the prescriber by the number of unique patients who were prescribed any drug by the prescriber in 2014. We elected to use patients to whom a physician had prescribed any drug as the denominator because it is the most specific cohort of patients at risk of being prescribed an antibiotic from that physician. Importantly, $98.1 \%$ of residents in long-term care facilities receive at least 1 drug prescription per year.

We calculated a prescriber's tendency to use prolonged treatment durations by dividing the number of treatment courses for antibiotics prescribed by the physician that exceeded 7 days by the number of treatment courses for antibiotics that they prescribed in 2014. We selected a cut-off of more than 7 days of treatment based on evidence that most bacterial infections can be treated with a duration of 7 days or less of antibiotics., ${ }^{6,34-38} \mathrm{We}$ considered consecutive prescriptions for the same antibiotic to be part of the same treatment course if the drug was prescribed by the same physician, to the same patient and there was no more than 3 days between prescriptions.

We calculated a prescriber's tendency to select the class of fluoroquinolone antibiotics by dividing the number of prescriptions for fluoroquinolones provided by the physician in 2014 by the number of treatment courses for antibiotics prescribed in 2014. Some researchers have recommended against prescribing fluoroquinolones for residents in long-term care because of the potential for resistance and toxicity. ${ }^{4,39}$ However, some guidelines have included fluoroquinolones as first-line treatment options in this population. ${ }^{40,41}$ We chose fluoroquinolones as 1 representative class of antibiotics to test whether prescriber-specific tendencies govern not only the decisions to start and prolong antibiotic treatment but also to test the choice of a specific agent.

\section{Characteristics of prescribers}

From the ICES physician database and other linked databases, we extracted an array of prescriber characteristics: demographic characteristics (age, sex), practice location (urban/rural), training history (country of graduation, years in practice), and volume of medications and antibiotics prescribed. Our primary predictors of interest were the prescribers' historical prescribing tendencies in the previous year (2013), including their tendencies to start antibiotics, use prolonged treatment durations and select fluoroquinolones. 
Table 1: Characteristics of prescribers and residents in long-term care facilities

\section{Characteristic}

Prescriber $(n=1695)$

No. of males (\%)

Age, yr

No. of prescribers with urban practices (\%)

No. of prescribers who graduated from Canadian medical schools (\%)

No. of general/family practitioners (\%)

No. of years in practice

No. of LTC prescriptions in 2014

No. of LTC prescriptions for antibiotics in 2014

No. of unique LTC residents as patients in 2014

No. of prolonged antibiotic treatments in 2014

No. of fluoroquinolone treatments in 2014

Resident ( $n=93$ 132)

Diabetes mellitus

Hypertension

Atherosclerotic heart disease

Stroke

Peripheral vascular disease

Dementia

Cancer

Chronic obstructive pulmonary disease/asthma

Parkinson disease

Gastrointestinal disease

Liver disease

Renal disease

Frailty

Dependent on assistance for

Transferring

Dressing

Eating

Toileting

Hygiene

Bowel incontinence

Bladder incontinence

Hearing impairment

Vision impairment

Urinary catheter

Dialysis

Intravenous drugs

Tracheostomy

Ventilator

Feeding tube
Variability across prescribers, median (IQR)*

$$
\begin{gathered}
1198(70.7) \\
57(47-64) \\
1243(73.3) \\
1356(80.0) \\
1650(97.3) \\
30(20-38) \\
7181(988-18924) \\
35(5-102) \\
17(3-43) \\
10(1-32) \\
9(1-29)
\end{gathered}
$$

$26(20-33)$

$66(58-73)$

15 (7-23)

$25(18-32)$

$5(0-9)$

$65(54-75)$

$8(3-13)$

$19(13-26)$

$6(2-10)$

$21(12-33)$

$0(0-1)$

$9(3-14)$

$60(48-71)$

$78(70-86)$

92 (86-98)

$36(27-47)$

$87(80-94)$

$92(86-98)$

$51(41-63)$

$75(67-83)$

$13(8-20)$

15 (9-22)

$4(0-7)$

$0(0-0)$

$2(0-5)$

$0(0-0)$

$0(0-0)$

$0(0-1)$

Note: IQR = interquartile range, $\mathrm{LTC}=$ long-term care.

*Unless otherwise specified. 


\section{Characteristics of residents}

We extracted detailed resident characteristics from the Continuing Care Reporting System Long-Term Care Database (ICES), which provides a mandatory, extensive, reliable and valid assessment of the comorbidity, functional status, care needs and indwelling devices used by residents in long-term care facilities. ${ }^{42}$ Details of this method are provided in our previous studies. ${ }^{6,12}$ The Resident Assessment Instrument - Minimum Data Set version 2.0 from the database ${ }^{43}$ is completed quarterly, and a full assessment is completed yearly; we used each resident's most recent full assessment as of Dec. 31, 2014.

\section{Statistical analysis}

We characterized the population of long-term care prescribers and variation in resident characteristics across prescribers. In particular, we sought to describe prescribers' tendencies to start antibiotics, to use prolonged durations and to select fluoroquinolone agents. By definition, we excluded physicians with no antibiotic prescriptions in 2014 for the analyses of prolonged durations and selection of fluoroquinolone agents.

For each physician, we examined whether each prescribing tendency in 2014 was correlated with the prescriber's tendency from 2013 by using graphical inspection of a scatter plot and calculation of a weighted Pearson correlation coefficient. We also examined cross-correlation between the 3 tendencies within 2014 by generating pairwise scatter plots and weighted correlation coefficients.

For each prescribing tendency, we created a funnel plot to examine whether interprescriber variation was greater than that expected by random chance. ${ }^{44}$ The funnel plot provides a graphical representation of variations in the prescribing tendencies $(y$-axis) as a function of prescriber volume ( $x$-axis). We generated control limits by using exact binomial confidence intervals (Cls), and prescribers were defined as extreme-low prescribers (below the -3 standard deviation [SD] control limit), low prescribers (below the -2SD limit), average prescribers (between the -2SD and +2 SD limits), high prescribers (above the +2 SD limit) and extreme-high prescribers (above the +3SD limit).

We used multivariable random-effects logistic regression models to assess the effect of prescriber-level characteristics on the likelihood of starting treatment with antibiotics, prolonged duration of treatment with antibiotics and selection of fluoroquinolones, and controlling for individual resident characteristics. The unit of analysis was the unique patient-physician pairing. We used a random-effects logistic regression model to regress outcomes (measured at the level of each unique patient-physician pairing) on characteristics of that pairing. Our model incorporated physician-specific random effects to allow outcomes within pairings that shared the same physician to be correlated with one another. We evaluated each physician-level characteristic in a separate model, with each of the models accounting for resident characteristics that potentially could be associated with the likelihood of needing treatment with antibiotics (including age, sex, 13 comorbidities (Table 1), dependence on assistance for each activity of daily living, incontinence status, sensory impairment and indwelling devices). ${ }^{4,12}$ Our main predictor of interest was the prescriber's historical prescribing tendency from the previous calendar year.
In sensitivity analyses, we extended historical prescribing patterns to a 3-year average (2011-2013). As an additional measure of heterogeneity in physician prescribing behaviour, we calculated a median odds ratio (OR), which indicates the median value of the ORs obtained when comparing the odds of antibiotic prescribing behaviour to a resident treated by a randomly selected physician with the odds of prescribing behaviour to a resident with identical characteristics treated by another randomly selected physician, where the physicians are ordered by prescribing tendencies. The median OR can be thought of as the median increase in the odds of occurrence of the antibiotic prescribing outcome that would arise when a resident moves from being treated by a lower- to a higher-tendency prescriber.

All analyses were conducted using SAS Enterprise Guide Version 6.1 (SAS Institute, Inc.).

\section{Ethics approval}

Research ethics approval was granted by the Research Ethics Board of Sunnybrook Health Sciences Centre. The study was conducted according to rigorous privacy safeguards in place at ICES.

\section{Results}

Our cohort of 1695 physicians were responsible for 24456286 drug prescriptions to 93132 residents in long-term care facilities. Prescribers were mostly male $(n=1198,70.7 \%)$, graduates of Canadian medical schools ( $n=1356,80.0 \%$ ), practising in urban settings ( $n=1243,73.3 \%)$ and trained as general/family practitioners $(n=1650,97.3 \%)$ (Table 1$)$.

More than half of the residents were frail (59.1\%) with substantial multimorbidities. Residents frequently had a history of dementia (63.2\%), diabetes mellitus (27.3\%), stroke (26.0\%) and urinary incontinence (74.5\%). Residents commonly depended on support for each of their activities of daily living, but few were dependent on indwelling devices, such as urinary catheters (5.5\%) (Table 1).

\section{Prescribing tendencies for antibiotics}

\section{Starting antibiotic treatment}

Prescribers were responsible for 219157 antibiotic prescriptions dispensed to $55 \%$ of residents $(n=51256)$ in long-term care facilities in 2014. Overall, a median of $44.9 \%$ (interquartile range [IQR] $32.1 \%-54.9 \%)$ of residents were treated with at least 1 antibiotic during the study year by prescribers. There was wide variability in tendency to start antibiotic treatment, with $25.0 \%$ of prescribers $(n=424)$ classified as low or extreme-low, and 16.0\% $(n=272)$ as high or extreme-high (Figure 1A).

\section{Prolonged duration of antibiotic treatment}

Across the 1521 out of 1695 (89.7\%) prescribers who wrote at least 1 antibiotic prescription in 2014, the median percentage of antibiotic prescriptions that were prolonged was $30.4 \%$ (IQR $18.5 \%-45.6 \%) ; 512(33.6 \%)$ of these prescribers were classified as low or extreme-low and 300 (19.7\%) as high or extreme-high users of prolonged durations (Figure 1B). 
A

- Extreme-low prescriber oLow prescriber Average prescriber $\circ$ High prescriber $\circ$ Extreme-high prescriber
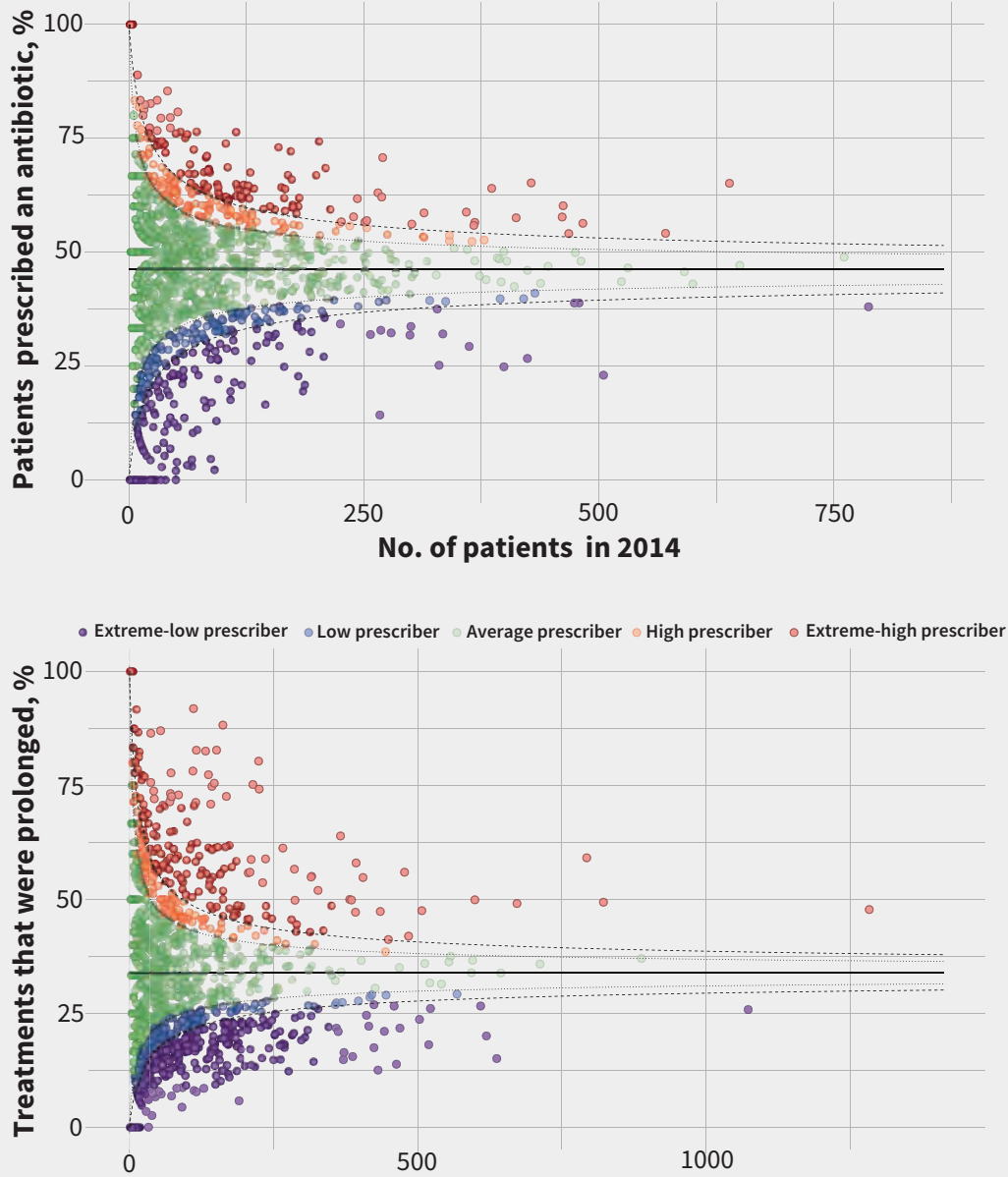

No. of antibiotic treatments in 2014

C

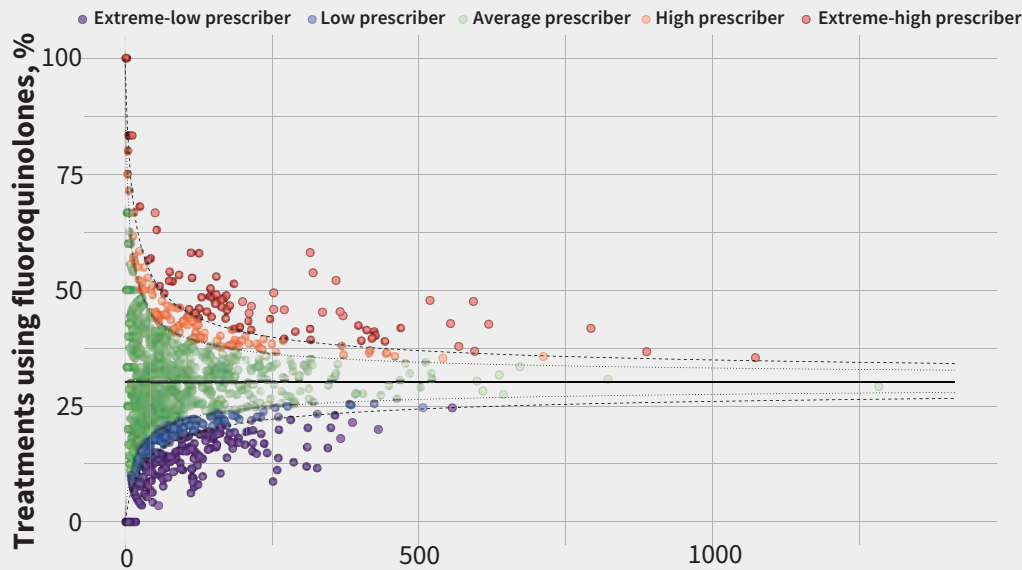

No. of antibiotic treatments in 2014

Figure 1: Funnel plots of prescriber tendencies. Overall population averages are denoted by a black line in each plot. Each prescriber is represented by an individual filled circle. (A) Prescriber tendency to start treatment with antibiotics as a percentage of their unique patients in long-term care facilities in 2014. Prescribing tendencies are classified into extreme-low, low, average, high or extreme-high initiators, based on where they fall compared with the control limits at 2SD (dotted line) and 3SD (broken line). (B) Prescriber tendency to use treatment durations that exceeded 7 days as a percentage of their overall antibiotic prescriptions to patients in long-term care facilities. Prescribing tendencies are classified into extreme-low, low, average, high or extreme-high use of prolonged treatment duration, based on where they fall compared with the control limits at 2SD (dotted line) and 3SD (broken line). (C) Prescriber tendency to select fluoroquinolones as a percentage of their overall antibiotic prescriptions to patients in long-term care facilities. Prescribing tendencies are classified into extreme-low, low, average, high or extremehigh users of fluoroquinolones, based on where they fall compared with the control limits at 2SD (dotted line) and 3SD (broken line). SD = standard deviation. 
A

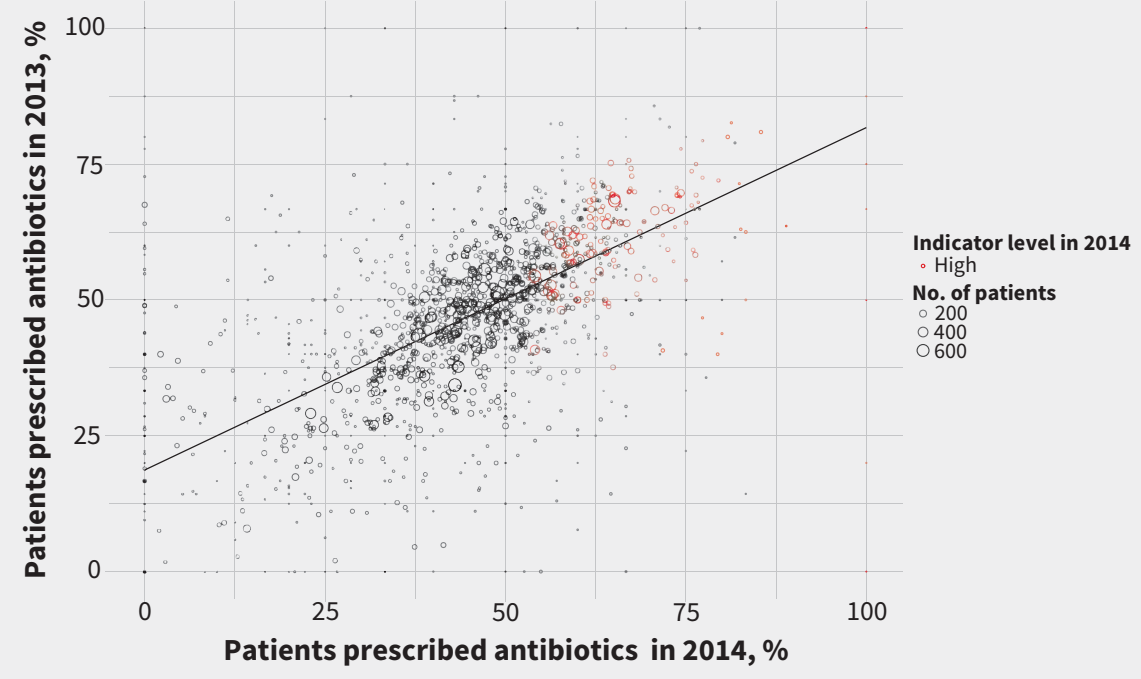

B

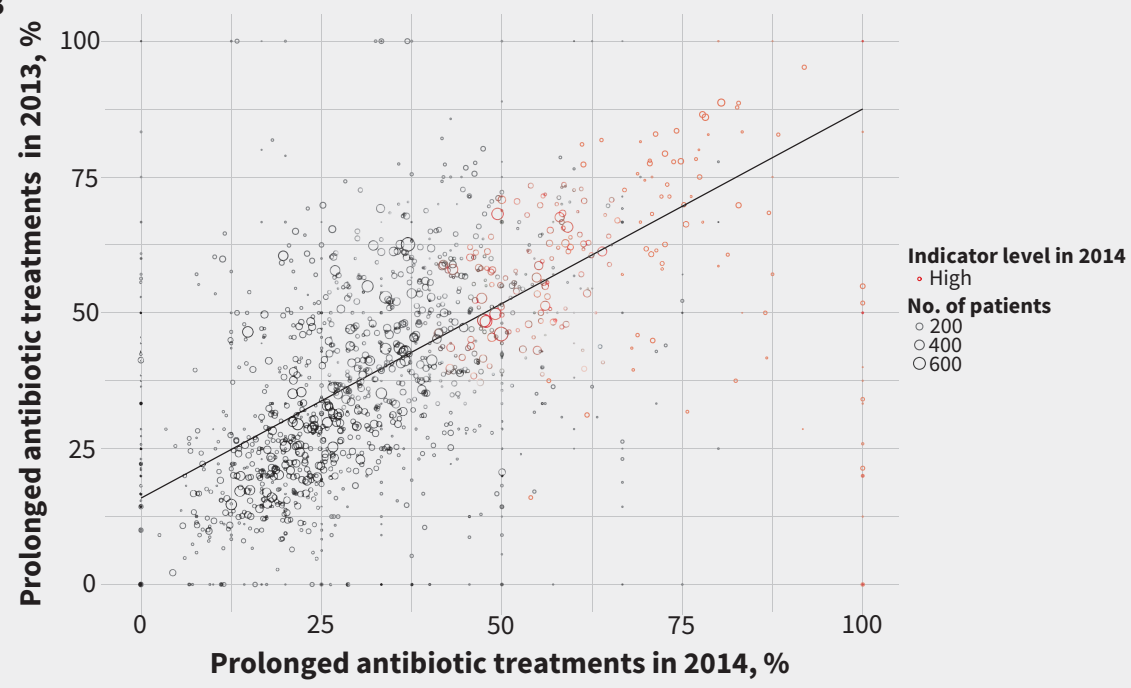

pe

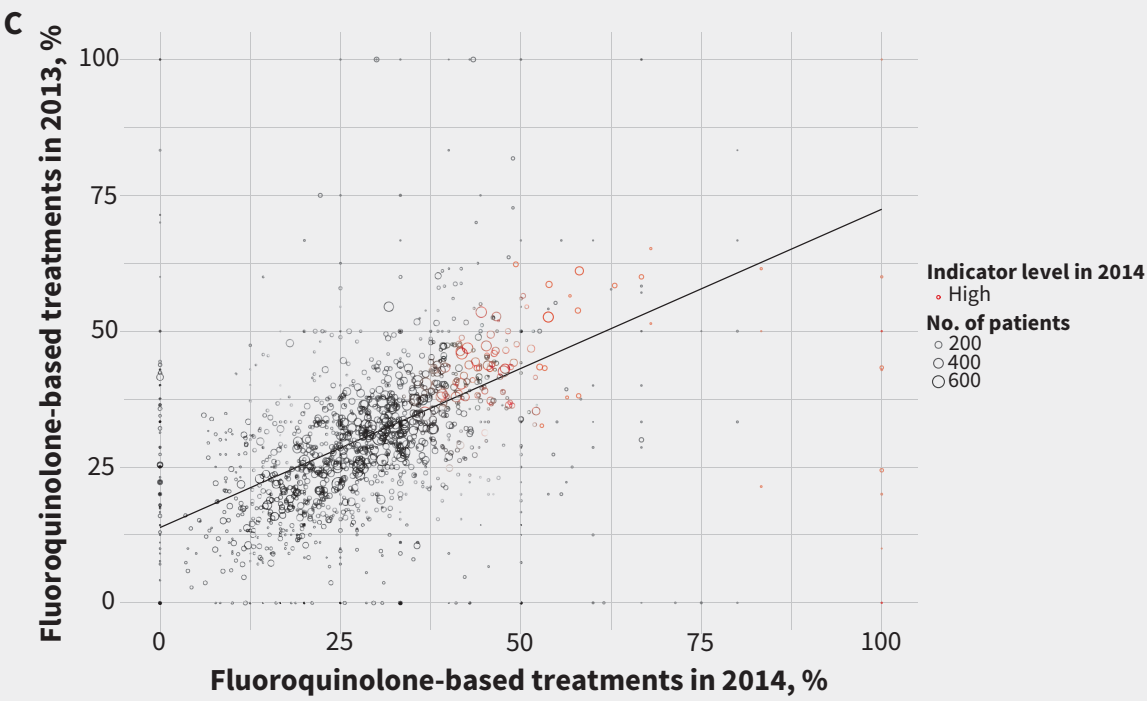

Figure 2: Correlations of prescriber tendencies in 2014 versus 2013. Each prescriber is represented by an individual black circle in the plots, with size proportional to clinical volume. (A) Prescriber tendency to start treatment with antibiotics. Prescribers shown as red circles were high or extreme-high starters in 2014. (B) Prescriber tendency to use prolonged durations of treatment with antibiotics. Prescribers shown as red circles were high or extreme-high users of prolonged durations in 2014. (C) Prescriber tendency to select fluoroquinolone antibiotics. Prescribers shown as red circles were high or extreme-high users of fluoroquinolones in 2014. 


\section{Selection of fluoroquinolone class}

Among the 1521 prescribers, the median use of fluoroquinolones was $27.3 \%$ (IQR $17.8 \%-37.2 \%) ; 429$ (28.2\%) of these prescribers were classified as low or extreme-low users and 196 (12.9\%) as high or extreme-high users of fluoroquinolones (Figure 1C).

\section{Correlation of current prescribing tendencies with historical patterns}

Tendency to start treatment with antibiotics in 2014 was strongly correlated with a prescriber's historical tendency to start antibiotic treatment (weighted Pearson correlation coefficient [PCC] 0.66 ; Figure $2 \mathrm{~A}$ ). Similarly, there was a strong correlation between current and historical tendencies to use prolonged durations of treatment (PCC 0.64; Figure 2B) and between the current and historical tendencies to select fluoroquinolones (PCC 0.58; Figure $2 \mathrm{C}$ ). Most prescribers who were extreme-high outliers in 2013 remained at least high outliers in 2014, including 102 of 153 $(66.7 \%)$ prescribers who were extreme-high to start antibiotic treatment, 143 of 242 (59.1\%) who were extreme-high users of prolonged durations and 61 of 103 (59.2\%) who were extremehigh in selection of fluoroquinolones.

\section{Cross-correlation of prescribing tendencies}

In contrast, there was weak to no within-year correlation of the 3 prescribing tendencies for antibiotics: start of treatment and use of prolonged duration (PCC +0.08 ), start of treatment and selection of fluoroquinolones (PCC +0.08 ), and use of prolonged duration and selection of fluoroquinolones (PCC -0.007) (Appendix 1, Supplemental Figures 1A, B and C, available at www.cmaj. ca/lookup/suppl/doi:10.1503/cmaj.161437/-/DC1).

\section{Multivariable logistic regression}

Distributions of resident demographics, comorbidities, frailty levels, functional status and device dependence were similar among prescribers described as extreme-high, high, average, low and extreme-low (data not shown). After accounting for residentlevel characteristics, medical graduates from outside Canada were slightly more likely to start treatment with antibiotics, and prescribers in rural areas were slightly less likely to select fluoroquinolones (Table 2). A prescriber's historical prescribing tendency in 2013 was the strongest physician predictor of current practice in 2014. For example, a prescriber who was described as extreme-high to start antibiotic treatment for the

Table 2: Effect of prescriber characteristics on prescribing decisions for antibiotics to individual residents in long-term care facilities, after accounting for resident-level characteristics

\begin{tabular}{|c|c|c|c|}
\hline Prescriber characteristic* & $\begin{array}{l}\text { Starting antibiotic } \\
\text { treatment, } \\
\text { OR }(95 \% \mathrm{Cl})\end{array}$ & $\begin{array}{l}\text { Use of prolonged } \\
\text { duration for treatment, } \\
\text { OR }(95 \% \mathrm{Cl})\end{array}$ & $\begin{array}{l}\text { Selection of } \\
\text { fluoroquinolone class } \\
\text { OR }(95 \% \mathrm{CI})\end{array}$ \\
\hline \multicolumn{4}{|l|}{ Age, yr } \\
\hline$<45$ & 1.00 (ref) & 1.00 (ref) & 1.00 (ref) \\
\hline $45-55$ & $1.05(0.95-1.17)$ & $1.04(0.90-1.21)$ & $0.92(0.82-1.03)$ \\
\hline $56-65$ & $1.04(0.94-1.15)$ & $1.11(0.96-1.28)$ & $1.02(0.91-1.13)$ \\
\hline$\geq 66$ & $0.97(0.87-1.08)$ & $1.36(1.15-1.59)$ & $0.98(0.87-1.11)$ \\
\hline \multicolumn{4}{|l|}{ Sex } \\
\hline Male & 1.00 (ref) & 1.00 (ref) & 1.00 (ref) \\
\hline Female & $0.99(0.92-1.07)$ & $0.81(0.73-0.91)$ & $0.95(0.87-1.03)$ \\
\hline \multicolumn{4}{|l|}{ Location } \\
\hline Urban & 1.00 (ref) & 1.00 (ref) & 1.00 (ref) \\
\hline Rural & $0.93(0.86-1.01)$ & $0.92(0.81-1.03)$ & $0.85(0.77-0.93)$ \\
\hline \multicolumn{4}{|l|}{ Place of graduation } \\
\hline Canada & 1.00 (ref) & 1.00 (ref) & 1.00 (ref) \\
\hline Outside Canada & $1.10(1.01-1.20)$ & $1.09(0.96-1.24)$ & $1.07(0.97-1.17)$ \\
\hline \multicolumn{4}{|c|}{ Historical prescribing pattern in 2013} \\
\hline Extreme-high & $1.87(1.70-2.06)$ & $2.24(2.01-2.48)$ & $1.80(1.61-2.01)$ \\
\hline High & $1.44(1.31-1.58)$ & $1.57(1.35-1.82)$ & $1.48(1.33-1.65)$ \\
\hline Average & 1.00 (ref) & 1.00 (ref) & 1.00 (ref) \\
\hline Low & $0.69(0.62-0.77)$ & $0.63(0.54-0.72)$ & $0.67(0.60-0.74)$ \\
\hline Extreme-low & $0.43(0.39-0.51)$ & $0.46(0.42-0.51)$ & $0.55(0.51-0.61)$ \\
\hline \multicolumn{4}{|c|}{$\begin{array}{l}\text { Note: } \mathrm{Cl}=\text { confidence interval, } \mathrm{OR}=\text { odds ratio, ref = reference category. } \\
\text { "Each prescriber characteristic was evaluated separately in a multivariable model that adjusted for the following resident characteristics: age; sex; diabetes mellitus; hypertension; } \\
\text { atherosclerotic heart disease; stroke; peripheral vascular disease; dementia; cancer; chronic obstructive pulmonary disease/asthma; Parkinson disease; gastrointestinal disease; liver } \\
\text { disease; renal disease; assistance with transferring, dressing, eating, toileting or hygiene; incontinence of bowel or bladder; impairment of hearing, vision or indwelling urinary } \\
\text { catheter; dialysis; tracheostomy; ventilator; and feeding tube. }\end{array}$} \\
\hline
\end{tabular}


previous year was more likely to start antibiotic treatment (adjusted OR 1.87, 95\% Cl 1.70-2.06) than a prior prescriber who was described as average (Table 2 ).

\section{Sensitivity analyses}

We extended the measure of historical prescribing tendencies to include average prescribing behaviour over the preceding 3 years (2011-2013) to address concerns about consistency in historical patterns of prescribing. The strong correlation we found between current and historical prescribing tendencies remained evident for the tendency to start treatment with antibiotics (PCC 0.48), tendency to use prolonged durations (PCC 0.66) and tendency to select fluoroquinolones (PCC 0.57). In multivariable regression, our use of a longer historical period only slightly attenuated the association of historical and current prescribing; a prescriber who was extreme-high to start antibiotics in the previous 3 years was still more likely to start antibiotics (adjusted OR 1.49, 95\% Cl 1.37-1.63).

After adjustment for resident and physician characteristics, a resident who moved to being treated by a prescriber with a higher tendency to start antibiotic treatment had a median OR of 1.49 of having an antibiotic prescription started during the year; a resident moving to treatment by a higher user of prolonged treatment durations would have a median OR of 1.65 for receiving prolonged treatment; and a resident moving to treatment by a higher user of fluoroquinolones would have a median OR of 1.46 for receiving a fluoroquinolone.

\section{Interpretation}

Our population-based analysis of physicians who regularly prescribe to residents in long-term care facilities found wide variability in prescribers' tendencies to start antibiotics, use prolonged treatment durations and select fluoroquinolones. These prescribing tendencies were consistent over time.

At the resident level, after controlling for resident characteristics, the likelihoods of an antibiotic being started, of a treatment being prolonged, or of a fluoroquinolone being selected were strongly predicted by the prescriber's historical tendencies.

This study builds upon our previous finding that prolonged durations of treatment with antibiotics are driven more by prescribers than residents, ${ }^{6}$ and shows that other pivotal decisions about antibiotic treatment (start of treatment and drug selection) were also driven by prescribers. Moreover, we showed that these tendencies were stable over time.

If prescribers have consistent and measurable tendencies for each of these domains of antibiotic prescribing, this suggests that stewardship interventions for antibiotics that are focused on individual prescribers are feasible on a large scale. Maximum benefit may be accrued by focusing attention on the prescribers that are historical outliers..$^{45}$ Our data suggest that the high outliers for starting treatment with antibiotics are not the same high outliers for duration or class selection. Therefore, different physician subgroups should be targeted for interventions aimed at each domain of prescribing behaviour. Given the extensive barriers to appropriate antimicrobial prescribing in long-term care facilities, ${ }^{4,14,15}$ some prescriber-level interventions may be labour-intensive or offer only short-lived benefits (e.g., prescriber education and detailing). Perhaps the most pragmatic, effective and scalable intervention would be peer comparison audit and feedback. Audit and feedback has shown potential to improve health care behaviours and is particularly effective for drug prescribing. ${ }^{46}$

The suggestion that decision-making about antibiotics might be prone to prescriber tendencies is plausible, because humans are "creatures of habit." Habits are "automatic responses to contextual cues, acquired through repetition of behaviour in the presence of these cues" and are more likely to form within consistent contexts. ${ }^{47}$ Many health care contexts, such as long-term care facilities, remain relatively constant over time and can lead to habit-forming behaviour. ${ }^{47}$ Similarly, the process of writing drug prescriptions is highly repetitive. Previous research has shown that prescribers may develop distinct prescription profiles (e.g., choosing generic versus trade name drugs ${ }^{48}$ or adopting newer agents ${ }^{49}$ ). Physicians typically choose from a short list of "evoked drugs" that are at the "top of their mind" and account for most of their prescriptions. ${ }^{50}$ Our study suggests that prescriber-level tendencies govern the 3 key aspects of antimicrobial prescribing. It is possible that similar prescriber tendencies are driving the geographic variation in outpatient prescribing of antibiotics to older adults seen in the United States. ${ }^{51-53}$

\section{Limitations}

Our study is subject to the limitations of health services research, but misclassification of antibiotic use is uncommon in the drug database (accuracy $99.7 \%$ v. pharmacy chart audits). ${ }^{33}$ We excluded prescribers who wrote less than 100 prescriptions in long-term care facilities in 2013 and 2014, therefore, we cannot be sure that our results are generalizable to occasional long-term care clinicians. However, a feasible stewardship intervention would need to target a finite number of prescribers, and our prescribers accounted for more than $80 \%$ of antibiotic prescriptions in long-term care facilities.

Although we adjusted for extensive resident characteristics, there is still the possibility that the consistent prescriber tendencies may not be completely governed by an internal locus of control but could also be subject to an external locus of control. This confounding by context could include unmeasured aspects of patient case mix in the facility, ranging from infection predisposition, to end-of-life care and to local patient expectations for antibiotic treatment, ${ }^{54}$ as well as unmeasured variability in local antimicrobial-resistant pathogens or patterns of service provision among the included general practitioners.

Our methods may have underestimated durations of antibiotic treatment in cases in which antibiotics were switched or streamlined during a course of treatment. An assessment of the appropriateness of each start of antibiotic treatment, duration and class selection is beyond the scope of a study of this size, given that the suspected or documented focus of infection is unavailable for most patients. ${ }^{32}$ However, previous research has consistently documented that $25 \%-75 \%$ of antibiotic treatments in long-term care facilities are inappropriate. ${ }^{4,5,55}$ 


\section{Conclusion}

The likelihood of a resident in a long-term care facility being started on an antibiotic, and the likelihood that it will be prolonged in duration or include a fluoroquinolone agent, are all highly dependent on the historical tendencies of the prescribing clinician. Given that antibiotic use in long-term care is often inappropriate, inappropriately prolonged or inappropriately selected, the quality of prescribing in long-term care facilities could benefit from prescriber-level interventions such as audit and feedback, which target physicians with measurable, historical practice patterns of antibiotic overuse or misuse.

\section{References}

1. Pakyz AL, Dwyer LL. Prevalence of antimicrobial use among United States nursing home residents: results from a national survey. Infect Control Hosp Epidemiol 2010;31:661-2.

2. D'Agata E, Mitchell SL. Patterns of antimicrobial use among nursing home residents with advanced dementia. Arch Intern Med 2008;168:357-62.

3. Mitchell SL, Shaffer ML, Loeb MB, et al. Infection management and multidrugresistant organisms in nursing home residents with advanced dementia. JAMA Intern Med 2014;174:1660-7.

4. Nicolle LE, Bentley DW, Garibaldi R, et al. Antimicrobial use in long-term-care facilities. SHEA Long-Term-Care Committee. Infect Control Hosp Epidemiol 2000;21:537-45.

5. Rotjanapan P, Dosa D, Thomas KS. Potentially inappropriate treatment of urinary tract infections in two Rhode Island nursing homes. Arch Intern Med 2011;171:438-43.

6. Daneman N, Gruneir A, Bronskill SE, et al. Prolonged antibiotic treatment in long-term care: role of the prescriber. JAMA Intern Med 2013;173:673-82.

7. Budnitz DS, Lovegrove MC, Shehab N, et al. Emergency hospitalizations for adverse drug events in older Americans. N Engl J Med 2011;365:2002-12.

8. Simor AE, Bradley SF, Strausbaugh LJ, et al. Clostridium difficile in long-termcare facilities for the elderly. Infect Control Hosp Epidemiol 2002;23:696-703.

9. Riggs MM, Sethi AK, Zabarsky TF, et al. Asymptomatic carriers are a potential source for transmission of epidemic and nonepidemic Clostridium difficile strains among long-term care facility residents. Clin Infect Dis 2007;45:992-8.

10. Bonomo RA. Multiple antibiotic-resistant bacteria in long-term-care facilities: an emerging problem in the practice of infectious diseases. Clin Infect Dis 2000; 31:1414-22.

11. Hujer AM, Bethel CR, Hujer KM, et al. Antibiotic resistance in the institutionalized elderly. Clin Lab Med 2004;24:343-61.

12. Daneman N, Bronskill SE, Gruneir A, et al. Variability in antibiotic use across nursing homes and the risk of antibiotic-related adverse outcomes for individual residents. JAMA Intern Med 2015;175:1331-9.

13. Nicolle LE. Antimicrobial stewardship in LTC facilities: What is effective? Antimicrob Resist Infect Control 2014;3:6.

14. Crnich CJ, Jump R, Trautner B, et al. Optimizing antibiotic stewardship in nursing homes: a narrative review and recommendations for improvement. Drugs Aging 2015;32:699-716.

15. Kistler CE, Sloane PD, Platts-Mills TF, et al. Challenges of antibiotic prescribing for assisted living residents: perspectives of providers, staff, residents, and family members. J Am Geriatr Soc 2013;61:565-70.

16. Schwartz DN, Abiad H, DeMarais PL, et al. An educational intervention to improve antimicrobial use in a hospital-based long-term care facility. J Am Geriatr Soc 2007;55:1236-42.

17. Pettersson E, Vernby A, Molstad S, et al. Can a multifaceted educational intervention targeting both nurses and physicians change the prescribing of antibiotics to nursing home residents? A cluster randomized controlled trial. J Antimicrob Chemother 2011;66:2659-66.

18. Linnebur SA, Fish DN, Ruscin JM, et al. Impact of a multidisciplinary intervention on antibiotic use for nursing home-acquired pneumonia. Am J Geriatr Pharmacother 2011;9:442-50.e1.
19. Loeb M, Brazil K, Lohfeld L, et al. Effect of a multifaceted intervention on number of antimicrobial prescriptions for suspected urinary tract infections in residents of nursing homes: cluster randomised controlled trial. BMJ 2005;331:669.

20. Zabarsky TF, Sethi AK, Donskey CJ. Sustained reduction in inappropriate treatment of asymptomatic bacteriuria in a long-term care facility through an educational intervention. Am J Infect Control 2008;36:476-80.

21. Rummukainen ML, Jakobsson A, Matsinen M, et al. Reduction in inappropriate prevention of urinary tract infections in long-term care facilities. Am J Infect Control 2012;40:711-4.

22. Monette J, Miller MA, Monette M, et al. Effect of an educational intervention on optimizing antibiotic prescribing in long-term care facilities. J Am Geriatr Soc 2007;55:1231-5.

23. Naughton BJ, Mylotte JM, Ramadan F, et al. Antibiotic use, hospital admissions, and mortality before and after implementing guidelines for nursing home-acquired pneumonia. J Am Geriatr Soc 2001;49:1020-4.

24. Jump RL, Olds DM, Seifi N, et al. Effective antimicrobial stewardship in a longterm care facility through an infectious disease consultation service: keeping a LID on antibiotic use. Infect Control Hosp Epidemiol 2012;33:1185-92.

25. Ivers N, Jamtvedt G, Flottorp S, et al. Audit and feedback: effects on professional practice and healthcare outcomes. Cochrane Database Syst Rev 2012;(6):CD000259.

26. Gruneir A, Bell CM, Bronskill SE, et al. Frequency and pattern of emergency department visits by long-term care residents - a population-based study. J Am Geriatr Soc 2010;58:510-7.

27. Tu JV, Donovan LR, Lee DS, et al. Effectiveness of public report cards for improving the quality of cardiac care: the EFFECT study: a randomized trial. JAMA 2009;302:2330-7.

28. Juurlink DN, Mamdani MM, Lee DS, et al. Rates of hyperkalemia after publication of the Randomized Aldactone Evaluation Study. N Engl J Med 2004;351:543-51.

29. Park-Wyllie LY, Juurlink DN, Kopp A, et al. Outpatient gatifloxacin therapy and dysglycemia in older adults. N Engl J Med 2006;354:1352-61.

30. Mamdani M, McNeely D, Evans G, et al. Impact of a fluoroquinolone restriction policy in an elderly population. Am J Med 2007;120:893-900.

31. Rochon PA, Stukel TA, Bronskill SE, et al. Variation in nursing home antipsychotic prescribing rates. Arch Intern Med 2007;167:676-83.

32. Daneman N, Gruneir A, Newman A, et al. Antibiotic use in long-term care facilities. J Antimicrob Chemother 2011;66:2856-63.

33. Levy AR, O'Brien BJ, Sellors C, et al. Coding accuracy of administrative drug claims in the Ontario Drug Benefit database. Can J Clin Pharmacol 2003;10:67-71.

34. Havey TC, Fowler RA, Daneman N. Duration of antibiotic therapy for bacteremia: a systematic review and meta-analysis. Crit Care 2011;15:R267.

35. Rafailidis PI, Pitsounis AI, Falagas ME. Meta-analyses on the optimization of the duration of antimicrobial treatment for various infections. Infect Dis Clin North Am 2009;23:269-76.

36. Sandberg T, Skoog G, Hermansson AB, et al. Ciprofloxacin for 7 days versus 14 days in women with acute pyelonephritis: a randomised, open-label and double-blind, placebo-controlled, non-inferiority trial. Lancet 2012;380:484-90.

37. Hepburn MJ, Dooley DP, Skidmore PJ, et al. Comparison of short-course (5 days) and standard (10 days) treatment for uncomplicated cellulitis. Arch Intern Med 2004;164:1669-74.

38. Tellier G, Niederman MS, Nusrat R, et al. Clinical and bacteriological efficacy and safety of 5 and 7 day regimens of telithromycin once daily compared with a 10 day regimen of clarithromycin twice daily in patients with mild to moderate community-acquired pneumonia. J Antimicrob Chemother 2004;54:515-23.

39. Hughes CM, Tunney MM. Improving prescribing of antibiotics in long-term care: Resistant to change? JAMA Intern Med 2013;173:682-3.

40. Mandell LA, Wunderink RG, Anzueto A, et al. Infectious Diseases Society of America/American Thoracic Society consensus guidelines on the management of community-acquired pneumonia in adults. Clin Infect Dis 2007;44(Suppl 2):S27-72.

41. Hooton TM, Bradley SF, Cardenas DD, et al. Diagnosis, prevention, and treatment of catheter-associated urinary tract infection in adults: 2009 International Clinical Practice Guidelines from the Infectious Diseases Society of America. Clin Infect Dis 2010;50:625-63.

42. Mor V. A comprehensive clinical assessment tool to inform policy and practice: applications of the minimum data set. Med Care 2004;42(Suppl4):III50-9. 
43. Hirdes JP, Fries BE, Morris JN, et al. Integrated health information systems based on the RAI/MDS series of instruments. Healthc Manage Forum 1999;12:30-40.

44. Spiegelhalter DJ. Funnel plots for comparing institutional performance. Stat Med 2005;24:1185-202.

45. Hallsworth M, Chadborn T, Sallis A, et al. Provision of social norm feedback to high prescribers of antibiotics in general practice: a pragmatic national randomised controlled trial. Lancet 2016;387:1743-52.

46. Ivers NM, Grimshaw JM, Jamtvedt G, et al. Growing literature, stagnant science? Systematic review, meta-regression and cumulative analysis of audit and feedback interventions in health care. J Gen Intern Med 2014;29:1534-41.

47. Nilsen P, Roback K, Brostrom A, et al. Creatures of habit: accounting for the role of habit in implementation research on clinical behaviour change. Implement Sci 2012;7:53.

48. Hellerstein JK. The importance of the physician in the generic versus tradename prescription decision. Rand J Econ 1998;29:108-36.
49. Lo-Ciganic WH, Gellad WF, Huskamp HA, et al. Who were the early adopters of dabigatran?: An application of group-based trajectory models. Med Care 2016;54:725-32.

50. de Jong JD, Groenewegen PP, Spreeuwenberg P, et al. Do decision support systems influence variation in prescription? BMC Health Serv Res 2009;9:20.

51. Zhang Y, Steinman MA, Kaplan CM. Geographic variation in outpatient antibiotic prescribing among older adults. Arch Intern Med 2012;172:1465-71.

52. Lee GC, Reveles KR, Attridge RT, et al. Outpatient antibiotic prescribing in the United States: 2000 to 2010. BMC Med 2014;12:96.

53. Hicks LA, Bartoces MG, Roberts RM, et al. US outpatient antibiotic prescribing variation according to geography, patient population, and provider specialty in 2011. Clin Infect Dis 2015;60:1308-16.

54. Mustafa M, Wood F, Butler CC, et al. Managing expectations of antibiotics for upper respiratory tract infections: a qualitative study. Ann Fam Med 2014;12:29-36.

55. Warren JW, Palumbo FB, Fitterman L, et al. Incidence and characteristics of antibiotic use in aged nursing home patients. J Am Geriatr Soc 1991;39:963-72.

\section{Competing interests: None declared.}

This article has been peer reviewed.

Affiliations: Institute for Clinical Evaluative Sciences (Daneman, Campitelli, Giannakeas, Bell, Maxwell, Austin, Bronskill), Toronto, Ont.; Sunnybrook Research Institute and Division of Infectious Diseases (Daneman), Sunnybrook Health Sciences Centre; Department of Medicine (Daneman, Morris, Bell); Institute of Health Policy, Management and Evaluation (Daneman, Bell, Austin, Bronskill); Sinai Health System (Morris, Bell); St. Michael's Hospital (Jeffs), University of Toronto, Toronto, Ont.; University of Waterloo (Maxwell), Waterloo, Ont.

Contributors: All of the authors contributed substantially to the study design, and interpretation or drafting of the manuscript, revised it criti- cally for intellectual content, gave final approval of the version to be published and agreed to be accountable for all aspects of the work.

Funding: Nick Daneman is supported by a Clinician Scientist Salary Award from the Canadian Institutes of Health Research (CIHR). Peter Austin is supported by a Career Investigator Award from the Heart and Stroke Foundation of Canada (Ontario office). Funding for this project was made available through grants from the Canadian Frailty Network (formerly Technology Evaluation in the Elderly Network) (no. SIG2014-M1) and CIHR (no. MOP-93642).

Disclaimer: This study was supported by the Institute for Clinical Evaluative Sciences (ICES), which is funded by an annual grant from the
Ontario Ministry of Health and Long-Term Care (MOHLTC). The opinions, results and conclusions reported in this paper are those of the authors and are independent from the funding sources. No endorsement by ICES or the Ontario MOHLTC is intended or should be inferred. Parts of this material are based on data and information compiled and provided by the Canadian Institute for Health Information (ClHI). However, the analyses, conclusions, opinions and statements expressed herein are those of the authors, and not necessarily those of $\mathrm{CIHI}$.

Accepted: Feb. 1, 2017

Correspondence to: Nick Daneman, nick. daneman@sunnybrook.ca 